\title{
Combined nanoscale KPFM characterization and device simulation for the evaluation of the MOSFET variability related to metal gate workfunction fluctuations
}

\author{
A. Ruiz, N. Seoane, S. Claramunt, A. García-Loureiro, M. Porti and M. Nafria \\ Version: accepted article
}

\section{How to cite:}

A. Ruiz, N. Seoane, S. Claramunt, A. García-Loureiro, M. Porti and M. Nafria (2019) Combined nanoscale KPFM characterization and device simulation for the evaluation of the MOSFET variability related to metal gate workfunction fluctuations. Microelectronic Engineering, 216, 111048.

Doi: https://doi.org/10.1016/i.mee.2019.111048

\section{Copyright information:}

(C) 2019 Published by Elsevier B.V. This manuscript version is made available under the CC-BY-NC-ND 4.0 license 


\title{
Combined nanoscale KPFM characterization and device simulation for the evaluation of the MOSFET variability related to metal gate workfunction fluctuations
}

\author{
A. Ruiz ${ }^{1}$, N. Seoane ${ }^{2}$, S. Claramunt ${ }^{1}$, A. García-Loureiro ${ }^{2}$, M. Porti ${ }^{1}$, M. Nafria ${ }^{1}$ \\ ${ }^{1}$ Electronic Engineering Department, Universitat Autònoma de Barcelona, Barcelona 08193, Spain \\ ${ }^{2}$ CITIUS, Universidade de Santiago de Compostela, Santiago de Compostela 15782, Spain \\ Electronic address: ana.ruiz@uab.cat
}

\section{Summary}

In this work, a more realistic approximation based on 2D nanoscale experimental data obtained on a metal layer is presented to investigate the impact of the metal gate polycrystallinity on the MOSFET variability. The nanoscale data (obtained with a Kelvin Probe Force Microscope, KPFM) were introduced in a device simulator to analyze the effect of a TiN metal gate work functions (WF) fluctuations on the MOSFET electrical characteristics. The results demonstrate that the device characteristics are affected not only by the WF fluctuations, but also their spatial distribution, which is specially relevant in very small devices. The effect on these characteristics of the spatial distribution on the gate area of such fluctuations is also evaluated.

\section{Introduction}

Extensive studies have been devoted to investigate variability sources affecting ultra-scaled MOSFET technologies, as Random Dopant Distributions, Line Edge Roughness, high-k dielectric polycrystallization [1-5], interface traps [6-8] and metal polycrystallization [9-11]. Charges captured in traps located at the semiconductor/insulator interface can lead to fluctuations in the threshold voltage $\left(\mathrm{V}_{\mathrm{T}}\right)$ and the on-current $[12,13]$ introducing, therefore, deviceto-device variability. Such $V_{T}$ fluctuations also depend on the position of the traps along the channel [13]. Regarding metal gates, the metal polycrystallization results in grains with different sizes and orientations, which have associated different WFs. The random distribution of grains (and the corresponding WF) results in $\mathrm{V}_{\mathrm{T}}$ variability (TVV) [9-11]. Some works have already analyzed the impact of the WF fluctuations on the variability of MOSFETs $[9,10]$. However, the statistical models that were used make assumptions that might not be representative of real devices.

In this work, the impact of metal polycrystallization on the electrical parameters of MOSFETs is studied, based on KPFM experimental WF maps. These results are compared to those obtained using the approximated WF distributions normally used in customary approaches for this kind of variability source. An analysis of the influence of the location of the WF fluctuations along the channel is also studied.

\section{Experimental set-up.}

A $100 \mathrm{~nm}$ thick TiN layer grown over a $\mathrm{HfO}_{2} / \mathrm{Si}$ substrate was used to obtain the experimental data. The layer was fabricated by continuous e-gun evaporation of metallic TiN. The TiN formation was ensured by passing the $\mathrm{Ti}$ atoms through a reactive nitrogen-enriched atmosphere (nitrogen partial pressure of $8 \times 10^{-4}$ mbars). X-Ray Diffraction (XRD) was used to determine the structure of the TiN layer. The XRD spectrum shows a polycrystalline structure with two peaks [14], that correspond to the [111] and [200] orientations [9]. being the [111] orientation dominant over the [200] one.

The nanoscale morphological and electrical properties of the TiN layer were measured with a Nano-Observer AFM from Concept Scientific Instruments. This technique allows bimodal single pass AM-KPFM measurements, so that it is possible to obtain during the same scan topographical and sample-tip contact potential difference (CPD) 2D maps with nanometer resolution $[15,16]$. Compared to a lift mode based AM-KPFM (normally with a worse resolution [17-19], our bimodal single pass AMKPFM can provide a similar resolution to that obtained with a FM-KPFM $[17,20]$ and minimizes cross-talk between topography and the CPD data $[18,21]$. Since the CPD image corresponds to the WF difference between the tip and the sample, assuming a constant value of the tip WF during the test, the measured CPD fluctuations can be related to the WF variations of the TiN layer. Therefore, CPD maps give information of the local value of the WF of the sample. KPFM images were obtained with highly doped Si tips to get a better resolution.

\section{Experimental results.}

Fig. 1a corresponds to a $520 \mathrm{~nm} \times 240 \mathrm{~nm}$ topographical image of the TiN layer. It shows a granular structure, where the Grains (Gs) are surrounded by grain boundaries (GBs), which correspond to the depressions in the image. Fig. 1b shows the measured WF map of the same surface region. Note that GBs tend to show lower WFs (WF < $4.1 \mathrm{eV}$ ) than nanocrystals.

We used the open-source software Gwyddion to identify the Gs and obtain the TiN granular pattern (Fig. 1c), where the GBs have been neglected. When only nanocrystals are considered, Gs with different maximum WF values are measured, as shown in Fig. 2 , which corresponds to a 2D-histogram that relates 
the maximum $\mathrm{WF}$ and the maximum height (with respect to the mean height value of the topographical image shown in Fig. 1a) of each grain in Fig. 1c. Note that although a continuous distribution of maximum WFs is obtained, WFs are mostly concentrated at $\sim 4.3$ $\mathrm{eV}$ and, with less frequency, around $\sim 4.5 \mathrm{eV}$, suggesting two predominant WFs and indicating a much higher number of nanocrystals with low WF than with high WF. This result, confirmed by XRD [14], is compatible with the presence of two grain orientations in the TiN layer ([111] and [200]) whose WFs are separated by $200 \mathrm{mV}$ [9] .
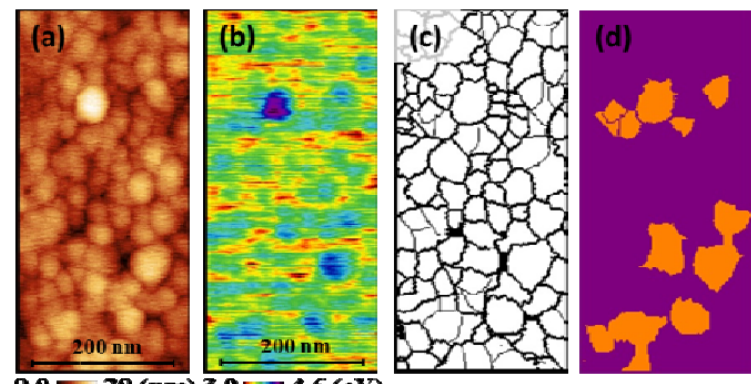

$0.0=20(\mathrm{~nm}) 3.9114 .6(\mathrm{eV})$

Fig. 1. Topography (a) and WF (b) maps obtained with KPFM on a TiN layer. Binary mask (c) of the grains identified in the topographical map and an example generated WF map (d) from the binary mask. Orange color corresponds to $4.5 \mathrm{eV}$ and purple color to $4.3 \mathrm{eV}$.

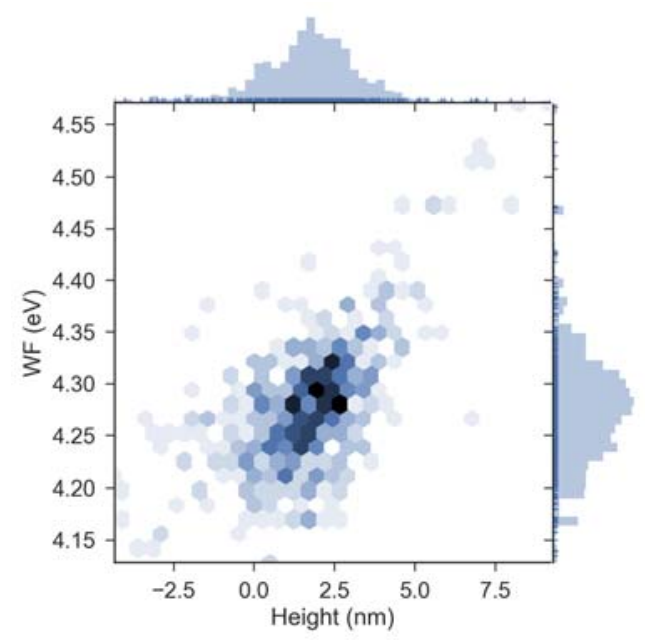

Fig. 2. 2D-histogram showing the maximum WF vs maximum height of the grains, obtained from Fig. 1c. In the $X$ axis, the height of each grain is determined with respect to the mean value of the topographical image (Fig. 1a).

The data obtained from KPFM images add new information on the properties of the polycrystalline metal layer, not taken into account in previous works. Besides the dispersion in the WF of the nanocrystals (not only two discrete values are measured), GBs with lower WF than Gs are also observed, which could also affect the variability of devices. Therefore, all these features should be taken into account when studying the MOSFET variability and when considering the WF fluctuations as variability source.

\section{Simulations}

In order to evaluate the device variability associated to the WF fluctuations of the TiN layer, different WF distributions representing the metal gate of a MOSFET were considered. WF maps as those shown in Fig. 1 have been divided to generate 100 non-overlapping $50 \mathrm{~nm} \times 50 \mathrm{~nm}$ gate $W F$ profiles. Next, these profiles were introduced in a 3D in-housebuilt drift-diffusion device simulator [22] as metal gate, to evaluate the device electrical characteristics of MOSFETs with a gate area of $50 \times 50 \mathrm{~nm}^{2}$. In order to compare the results obtained with our methodology, based on nanoscale experimental data, with those shown in the literature, we proposed the study of two cases: (i) the actual WF distribution (as in Fig. 1b, with GBs and a continuous distribution of Gs WF), and (ii) an approximated WF distribution, with two discrete values of the WF, as in Fig. 1d. Note that the last is the customary approach for this kind of TVV source [9]. To generate the approximated WF maps (Fig. 1d), the grain pattern in Fig. $1 \mathrm{c}$ is the starting point. We generated an approximated WF maps where GBs have been neglected and, for the Gs in Fig. 1c, two discrete and constant values, corresponding to the [111] and [200] orientations (confirmed from XRD analysis), were only considered. We have assigned $4.3 \mathrm{eV}$ to the nanocrystals with WF lower than $4.4 \mathrm{eV}$ and $4.5 \mathrm{eV}$ to those with higher values, leading to WF maps as that shown in Fig. 1d. The MOSFET behavior for devices with metal gate WF distributions obtained from the WF maps as in Fig. 1b and 1d have been compared.
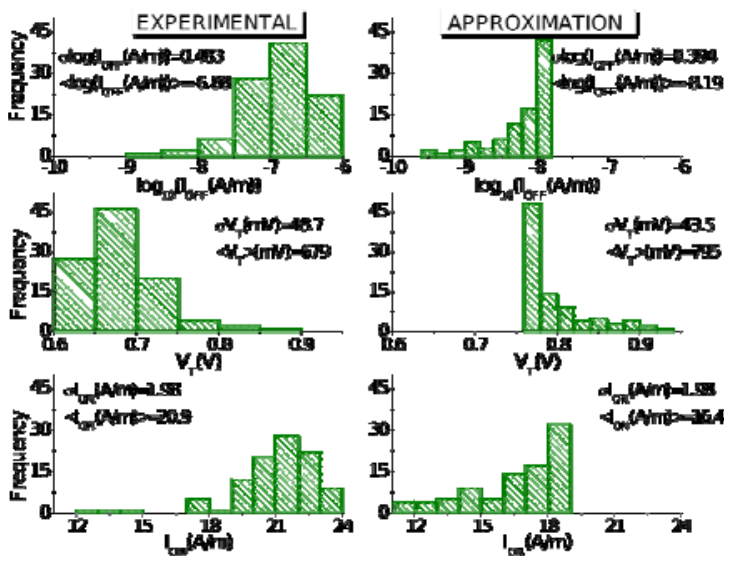

Fig. 3. Distributions of IOFF (top), $V_{T}$ (middle) and ION (bottom) related to $W_{F}$ fluctuations in $50 \mathrm{~nm} \times 50 \mathrm{~nm} \mathrm{Si}$ MOSFETs (at a drain bias of $50 \mathrm{mV}$ ) when using actual WF data (left column) an approximated WF (right column) from maps as those shown in Fig. $1 b$ and $1 d$ respectively. The mean value $(<>)$ and standard deviation $(\sigma)$ of the statistical ensembles are shown.

Fig. 3 shows the off-current $\left(\mathrm{I}_{\mathrm{OFF}}\right)$, threshold voltage $\left(\mathrm{V}_{\mathrm{T}}\right)$ and on-current $\left(\mathrm{I}_{\mathrm{ON}}\right)$ distributions obtained for these two cases. Note that, the approximate case (i. e., disregarding the GBs and the WF continuous distribution), in the right column, 
leads to $\mathrm{a} \sim 10 \%$ and $\sim 18 \%$ reduction in $\sigma \mathrm{V}_{\mathrm{T}}$ and $\sigma \log \left(\mathrm{I}_{\mathrm{OFF}}\right)$, respectively, and to a considerable shift $\left(\sim 0.12 \mathrm{~V}\right.$ in the case of the $\left.\mathrm{V}_{\mathrm{T}}\right)$ in the mean value of the distributions.

To better understand these results, the effect of the spatial location of the Gs in the MOSFET metal gate has also been evaluated. As an example, Fig. 4 shows the gate of two devices in which only one nanocrystal has been included. The average WF of the grains is $4.3 \mathrm{eV}$ for grain (a) and $4.18 \mathrm{eV}$ for grain (b). Since, in this section, we want to evaluate only the impact on $\mathrm{V}_{\mathrm{T}}$ of the position of the grains in the channel, the WF of the portion of the gate not covered by the nanocrystal has been set to same average value, that is, $4.3 \mathrm{eV}$ for Fig. $4 \mathrm{a}$ and $4.18 \mathrm{eV}$ for Fig. $4 \mathrm{~b}$. The $\mathrm{G}$ has been swept along the device gate, from the source end $(X=0 \mathrm{~nm})$ to the drain end $(X=50 \mathrm{~nm})$, and for each position of the grain, the device was simulated and the corresponding $\mathrm{V}_{\mathrm{T}}$ extracted. Fig. 5 shows the $\mathrm{V}_{\mathrm{T}}$ variation with respect to the homogeneous case when the grain position is swept, at both low and high drain biases. Note that, although the distribution of WF is the same in all cases, the specific location of the $G$ may have a different impact on the $V_{T}$ values (depending on its position along L), leading to both positive and negative threshold voltage shifts with respect to the grain average WF value. In addition, the device is clearly more sensitive to WF variations at the source end than at the drain end (as seen in Fig. 5 for both grains). At the drain end, the presence of the grain does not have any influence on $\mathrm{V}_{\mathrm{T}}$. The drain bias also has an influence on the TVV, as seen on Fig. 5. However, when the grains were swept along the ydirection (i.e. along the width of the gate area), no significant changes are observed in $\mathrm{V}_{\mathrm{T}}$.
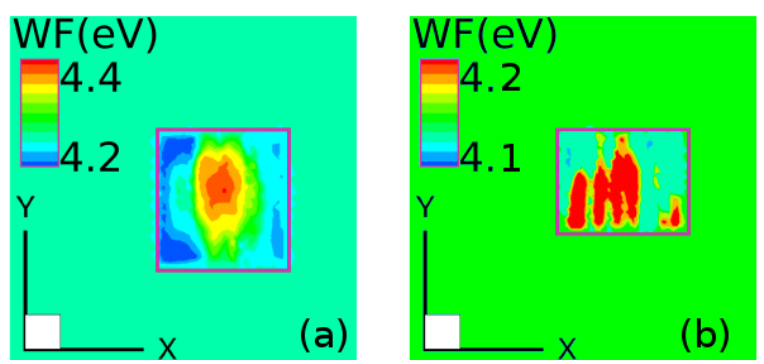

Fig. 4. Two examples of isolated nanocrystals (enclosed in pink rectangles), with average $W F$ values $\langle W F>$ of $4.3 \mathrm{eV}$, grain (a), and 4.18, grain (b). The larger green squares show the real dimensions of the $50 \times 50 \mathrm{~nm}$ device gate.

Therefore, the results demonstrate that, not only the WF values of the grains in the metal gate can impact on the MOSFET variability, but also $\mathrm{V}_{\mathrm{T}}$ depends on how those fluctuations are distributed along the channel direction. This dependence of $V_{T}$ on the $G$ position can be explained taking into account the impact of the WF fluctuations on the electrostatics of the device. Depending on the G location, the potential distribution in the channel is affected differently, leading to variations of $\mathrm{V}_{\mathrm{T}}$. A similar effect was observed for other sources of variability, as is the case of interface traps in ultra-scaled MOSFETs [6].
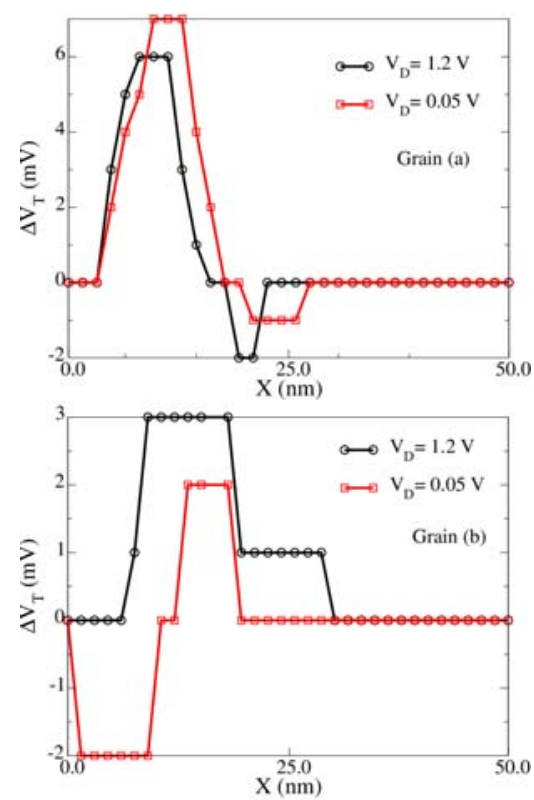

Fig. 5. $V_{T}$ spatial sensitivity to a TiN grain WF fluctuations when its position is swept along the channel of the Si MOSFET at both low and high drain biases like those shown in Fig $4 a$ and $4 b$ have seen considered for the simulation. $\Delta V_{T}$ is calculated as the difference between the $V_{T}$ of a device with a gate WF equal to the $\langle W F>$ of the $G$ and the $V_{T}$ of the device that includes the nanocrystal.

\section{Conclusion}

When analyzing the impact of the WF fluctuations of polycrystalline metal gates on the MOSFET variability, the presence of GBs and the WF continuous distribution of the Gs need to be considered to obtain more realistic data. Moreover, the device characteristics and variability are also affected by the spatial distribution of such fluctuations: in small devices, where few nanocrystals may be present in the gate area, their location should also be considered in order to correctly estimate the variability at device level.

\section{Acknowledgments}

This work has been partially supported by the Spanish AEI and ERDF (TEC2016-75151-C3-1-R, TEC2014-53909-REDT and RYC-2017-23312).

\section{References}

[1] K. Shubhakar, K.L. Pey, S.S. Kushvaha, S.J. O’Shea, N. Raghavan, M. Bosman, M. Kouda, K. Kakushima, H. Iwai, Grain boundary assisted degradation and breakdown study in cerium oxide gate dielectric using scanning tunneling microscopy, Appl. Phys. Lett. 98 (2011) 072902. doi:10.1063/1.3553190.

[2] V. Iglesias, M. Porti, M. Nafría, X. Aymerich, P. Dudek, T. Schroeder, G. Bersuker, Correlation between the nanoscale electrical and morphological properties of crystallized hafnium oxide-based metal oxide 
semiconductor structures, Appl. Phys. Lett. 97 (2010) 262906. doi:10.1063/1.3533257.

C. Couso, M. Porti, J. Martin-Martinez, A.J. GarciaLoureiro, N. Seoane, M. Nafria, Local Defect Density in Polycrystalline High-k Dielectrics: CAFM-Based Evaluation Methodology and Impact on MOSFET Variability, IEEE Electron Device Lett. 38 (2017) 637640. doi:10.1109/LED.2017.2680545.

[4] O. Pirrotta, L. Larcher, M. Lanza, A. Padovani, M. Porti, M. Nafría, G. Bersuker, Leakage current through the poly-crystalline HfO2: Trap densities at grains and grain boundaries, J. Appl. Phys. 114 (2013) 134503 doi: $10.1063 / 1.4823854$

[5] K. Murakami, M. Rommel, B. Hudec, A. Rosová, K. Hušeková, E. Dobročka, R. Rammula, A. Kasikov, J.H. Han, W. Lee, S.J. Song, A. Paskaleva, A.J. Bauer, L. Frey, K. Fröhlich, J. Aarik, C.S. Hwang, Nanoscale Characterization of $\mathrm{TiO}_{2}$ Films Grown by Atomic Layer Deposition on $\mathrm{RuO}_{2}$ Electrodes, ACS Appl. Mater. Interfaces. 6 (2014) 2486-2492. doi:10.1021/am4049139.

[6] V. Velayudhan, J. Martin-Martinez, R. Rodriguez, M. Porti, M. Nafria, X. Aymerich, C. Medina, F. Gamiz, TCAD simulation of interface traps related variability in bulk decananometer mosfets, 2014 5th Eur. Work. C. Var. VARI 2014. (2014) doi:10.1109/VARI.2014.6957078.

S.M. Amoroso, L. Gerrer, S. Markov, F. Adamu-Lema, A. Asenov, Comprehensive statistical comparison of RTN and BTI in deeply scaled MOSFETs by means of $3 \mathrm{D}$ atomistic simulation, Eur. Solid-State Device Res. Conf. (2012) 109-112.

doi:10.1109/ESSDERC.2012.6343345.

[8] B. Kaczer, T. Grasser, P.J. Roussel, J. Franco, R. Degraeve, L.A. Ragnarsson, E. Simoen, G. Groeseneken, $\mathrm{H}$. Reisinger, Origin of NBTI variability in deeply scaled pFETs, IEEE Int. Reliab. Phys. Symp. Proc. (2010) 2632. doi:10.1109/IRPS.2010.5488856.

H.F. Dadgour, K. Endo, V.K. De, K. Banerjee, Grainorientation induced work function variation in nanoscale metal-gate transistors - Part II: Implications for process, device, and circuit design, IEEE Trans. Electron Devices. 57 (2010) 2515-2525. doi:10.1109/TED.2010.2063270.

[10] N.M. Idris, A. Brown, J. Watling, A. Asenov, Simulation Study of Workfunction Variability in MOSFETs with Polycrystalline Metal Gates, Ultim. Integr. Silicon. 57 (2010) 165-168. http://eprints.gla.ac.uk/45013/.

[11] K. Ohmori, T. Matsuki, D. Ishikawa, T. Morooka, T. Aminaka, Y. Sugita, T. Chikyow, K. Shiraishi, Y. Nara, K. Yamada, Impact of additional factors in threshold voltage variability of metal/high-k gate stacks and its reduction by controlling crystalline structure and grain size in the metal gates, Tech. Dig. - Int. Electron Devices Meet. IEDM. (2008). doi:10.1109/IEDM.2008.4796707.
R. Rodriguez, M. Nafria, X. Aymerich, C. Marquez, F. Gamiz, Threshold voltage and on-current Variability related to interface traps spatial distribution, Eur. SolidState Device Res. Conf. 2015-Novem (2015) 230-233. doi:10.1109/ESSDERC.2015.7324756.

[13] V. Velayudhan, F. Gamiz, J. Martin-Martinez, R Rodriguez, M. Nafria, X. Aymerich, Influence of the interface trap location on the performance and variability of ultra-scaled MOSFETs, Microelectron. Reliab. 53 (2013) 1243-1246. doi:10.1016/j.microrel.2013.07.052.

[14] A. Ruiz, N. Seoane, S. Claramunt, A. Garcia-Loureiro, M. Porti, C. Couso, J. Martin-Martinez, M. Nafria, Workfunction fluctuations in polycrystalline TiN observed with KPFM and their impact on MOSFETs variability, Appl. Phys. Lett. 114 (2019) 093502. doi:10.1063/1.5090855.

[15] V. Iglesias, M. Lanza, K. Zhang, A. Bayerl, M. Porti, M Nafra, X. Aymerich, G. Benstteter, Z.Y. Shen, G. Bersuker, Degradation of polycrystalline $\mathrm{HfO}_{2}$-based gate dielectrics under nanoscale electrical stress, Appl. Phys. Lett. 99 (2011) 103510. doi:10.1063/1.3637633.

[16] T. Berthold, G. Benstetter, W. Frammelsberger, R. Rodríguez, M. Nafría, Nanoscale characterization of copper oxide films by Kelvin Probe Force Microscopy, Thin Solid Films. 584 (2015) 310-315. doi:10.1016/j.tsf.2015.01.071.

[17] J.L. Garrett, D. Somers, J.N. Munday, The effect of patch potentials in Casimir force measurements determined by heterodyne Kelvin probe force microscopy, J. Phys. Condens. Matter. 27 (2015) 214012. doi:10.1088/0953$8984 / 27 / 21 / 214012$.

[18] S.A. Burke, J.M. Ledue, Y. Miyahara, J.M. Topple, S. Fostner, P. Grütter, Determination of the local contact potential difference of PTCDA on $\mathrm{NaCl}$ : Acomparison of techniques, Nanotechnology. 20 (2009) 264012. doi:10.1088/0957-4484/20/26/264012.

[19] T. Glatzel, S. Sadewasser, M.C. Lux-Steiner, Amplitude or frequency modulation-detection in Kelvin probe force microscopy, Appl. Surf. Sci. 210 (2003) 84-89. doi:10.1016/S0169-4332(02)01484-8

[20] T. Ouisse, F. Martins, M. Stark, S. Huant, J. Chevrier, Signal amplitude and sensitivity of the Kelvin probe force microscopy, Appl. Phys. Lett. 88 (2006) 043102. doi:10.1063/1.2168251.

[21] G. Li, B. Mao, F. Lan, L. Liu, Practical aspects of singlepass scan Kelvin probe force microscopy, Rev. Sci. Instrum. 83 (2012) 113701. doi:10.1063/1.4761922.

[22] A.J. Garcia-Loureiro, N. Seoane, M. Aldegunde, R. Valin, A. Asenov, A. Martinez, K. Kalna, Implementation of the density gradient quantum corrections for 3-D simulations of multigate nanoscaled transistors, IEEE Trans. Comput. Des. Integr. Circuits Syst. 30 (2011) 841851. doi:10.1109/TCAD.2011.2107990. 